\title{
A Precise Description of Inorganic Nanoparticles in HRTEM Micrographs
}

\author{
D. Groom ${ }^{1}$, S. Rasouli², K. Yu ${ }^{2}$, A. Bovik ${ }^{3}$, P.J. Ferreira ${ }^{2}$ \\ 1. Chemical Engineering, University of Texas at Austin, Austin, TX \\ 2. Materials Science and Engineering Program, University of Texas at Austin, Austin, TX \\ 3. Electrical and Computer Engineering, University of Texas at Austin, Austin, TX
}

Nanoparticles (NPs) play an important role in a number of technologies, and many of their properties show a strong dependence on size and shape (i.e., their morphology) [4], [5], [9],[11]. There are numerous analytical methods used to characterize the morphology of NPs. Among these, transmission electron microscopy (TEM) represents a highly attractive option, primarily because it is the only analytical technique that directly allows for real space visualization of NPs [7]. However, subsampling presents a large source of uncertainty for this method of studying NP size, as many particle size distributions (PSDs) often represent data from a sample size on the order of 100 [8]. The primary reason for subsampling is that the complexity of TEM micrographs often precludes automated segmentation and sizing of NPs [10]. The need to manually segment NPs in TEM micrographs represents a bottleneck that must be overcome to address the crucial problem of subsampling. Sadly, there has been little work to date on this subject in the microscopy literature.

Figure 1 depicts the segmentation and classification tasks associated with sizing individual NPs in TEM micrographs. The first task is to identify the background of the image, which is colored red in the second image of Figure 1 [12]. Error can be introduced into the analysis when regions of the background are included in the sizing analysis as individual nanoparticles, so background exclusion is key to minimizing error [2]. The next task is to identify regions of the micrograph that are associated with NPs but that we want to exclude, which are colored green in the second image of Figure 1. Failure to exclude such regions represents a key source of error associated with this task. The final step is to identify individual NPs, which are colored blue in the second image of Figure 1. Failure to identify individual nanoparticles further exacerbates the problem of subsampling.

Common approaches for particle picking of inorganic nanoparticles in HRTEM micrographs fall into the classes of either global or local thresholding [3]. Numerous studies propose using one or the other of these approaches, but none quantify the error associated with using these thresholding approaches as compared to manual segmentation [1]. This study proposes an algorithm that hybridizes local thresholding with both texture and boundary information to yield a precise description of inorganic nanoparticles in HRTEM micrographs. Figure 2 contains the images of the three types of information that constitute this hybrid particle picking algorithm. To quantify the error associated with using this algorithm, we apply it along with global and local thresholding to a database of 100 images of Pt nanoparticles in the cathode of a proton exchange membrane fuel cell. Compared to the baseline of global thresholding, local thresholding is found to decrease false negatives by $77 \%$ and increases the number of individual particles segmented from regions of particle overlap by $95 \%$. However, doing so comes at a cost; local thresholding is found to increase false positives by $111 \%$. On the other hand, the proposed algorithm yields an ideal result, decreasing false negatives by $100 \%$, decreasing false positives by $85 \%$, and increasing individual particles segmented from regions of particle overlap by $253 \%$. Figure 3 contains some examples of these segmentation results. 


\section{References:}

[1] A. S. Abutaleb, Computer Vision, Graphics, and Image Processing, vol. 47, no. 1, pp. 22-32, Jul. 1989.

[2] C. Adams, H. Benesi, and R. Meisenheimer, JOURNAL OF CATALYSIS, vol. 1, no. 4, pp. 336-344, Aug. 1962.

[3] S. Al-amri, N. Kalyankar, and K. D, Journal of Computing, vol. 2, no. 5, May 2010.

[4] R. Boyd and A. Cuenat, Journal of Nanoparticle Research, vol. 13, no. 1, pp. 105-113, Jan. 2011.

[5] M.-C. Daniel and D. Astruc, Chemical Reviews, vol. 104, no. 1, pp. 293-346, Dec. 2003.

[6] L. Gontard, D. Ozkaya, and R. Dunin-Borkowsi, Ultramicroscopy, vol. 111, no. 2, pp. 101-106, Jan. 2011.

[7] C. Laramy, ACS nano, vol. 9, no. 12, pp. 12488-12495, Dec. 2015.

[8] S. U. Lee, S. Yoon Chung, and R. H. Park, Computer Vision, Graphics, and Image Processing, vol. 52, no. 2, pp. 171-190, Nov. 1990.

[9] M. Reetz et al, THE JOURNAL OF PHYSICAL CHEMISTRY B, vol. 104, no. 37, pp. 8779-8781, Aug. 2000.

[10] F. Samopa and A. Asano, IJCSNS International Journal of Computer Science and Network Security, vol. 9, no. 4, pp. 292-299, Apr. 2009.

[11] M. Gaumet et al, European Journal of Pharmaceutics and Biopharmaceutics, vol. 69, no. 1, pp. 1-9, May 2008.

[12] L.-K. Huang and M.-J. J. Wang, Pattern Recognition, vol. 28, no. 1, pp. 41-51, Jan. 1995.

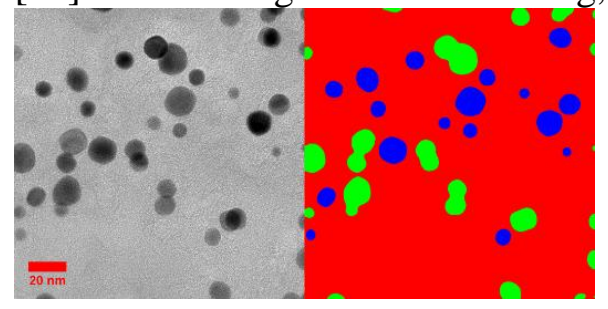

Figure 1. Selection of individual nanoparticles in HRTEM micrographs

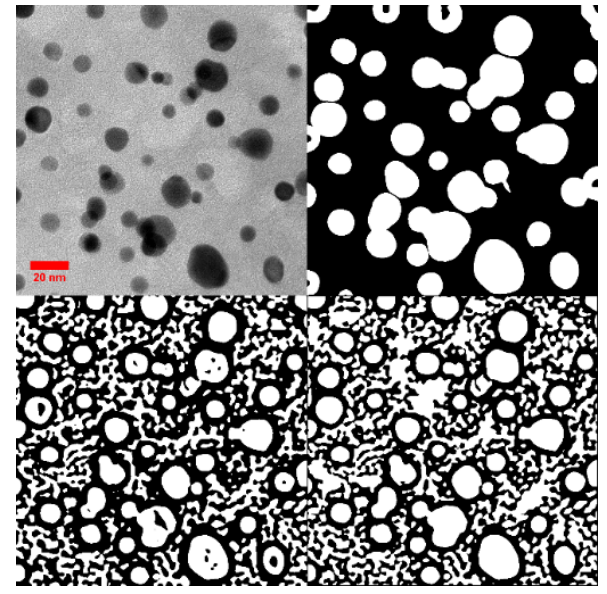

Figure 2. Elements of the proposed algorithm.

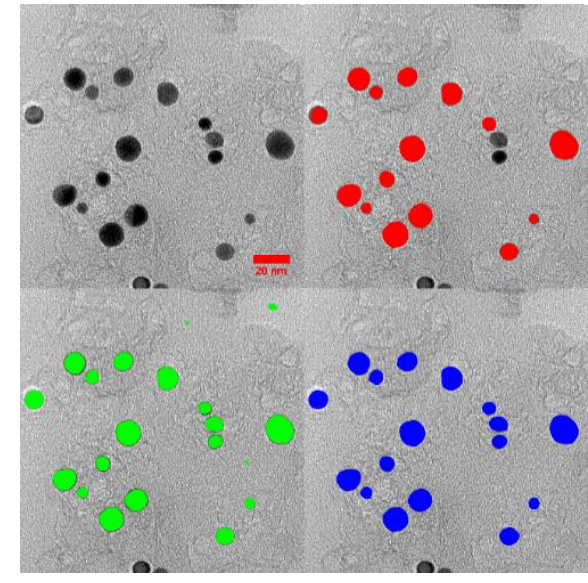

Figure 3. Segmentation results: global thresholding (red), local thresholding (green), the proposed hybrid algorithm. 